\title{
Functional Imaging Reveals Working Memory and Attention Interact to Produce the Attentional Blink
}

\author{
Stephen J. Johnston $^{1,2}$, David E. J. Linden ${ }^{2}$, and Kimron L. Shapiro ${ }^{2}$
}

\begin{abstract}
If two centrally presented visual stimuli occur within approximately half a second of each other, the second target often fails to be reported correctly. This effect, called the attentional blink (AB; Raymond, J. E., Shapiro, K. L., \& Arnell, K. M. Temporary suppression of visual processing in an RSVP task: An attentional blink? Joumal of Experimental Psychology, Human Perception and Performance, 18, 849-860, 1992], has been attributed to a resource "bottleneck," likely arising as a failure of attention during encoding into or retrieval from visual working memory
\end{abstract}

\section{INTRODUCTION}

The attentional blink (AB) reveals humans' limited ability to deploy attention to two successive targets presented within a short temporal interval. Traditionally, the $\mathrm{AB}$ is studied by having participants attempt to identify or detect two masked targets, often appearing as part of a rapid serial visual presentation (RSVP; cf., Raymond, Shapiro, \& Arnell, 1992). The stimuli, often letters or digits, are typically presented at a rate of between 6 and 20 items per second. The behavioral finding is that, if the second target (T2) appears within approximately $500 \mathrm{msec}$ of the first (T1), it often fails to be reported correctly (e.g., Chun \& Potter, 1995; Raymond et al., 1992), often with the participants having no conscious recollection of the second target ever having appeared (Sergent \& Dehaene, 2004).

A single account of the $A B$ is still heavily contested. For example, the $\mathrm{AB}$ has been attributed to retrieval interference between items in WM (Shapiro, Raymond, \& Arnell, 1994), failure to consolidate items into WM (Chun \& Potter, 1995), over-investment of resources to the RSVP stream before the first target (Olivers \& Nieuwenhuis, 2006), and disruption of target-specific attentional filters (Di Lollo, Kawahara, Ghorashi, \& Enns, 2005). Despite differences in accounts of the $\mathrm{AB}$, the majority converge to posit a failure in the deployment of attentional resources at the stage of visual working memory (WM) ${ }^{1}$ Specifically, concurrent processing of the first target deprives the second

${ }^{1}$ Brunel University, Uxbridge, UK, ${ }^{2}$ Bangor University
(WM). Here we present participants with a hybrid WM-AB study while they undergo fMRI to provide insight into the neural underpinnings of this bottleneck. Consistent with a WM-based bottleneck account, fronto-parietal brain areas exhibited a WM load-dependent modulation of neural responses during the $\mathrm{AB}$ task. These results are consistent with the view that WM and attention share a capacity-limited resource and provide insight into the neural structures that underlie resource allocation in tasks requiring joint use of WM and attention. target of limited attentional resources, either during retrieval from WM (Shapiro, Arnell, \& Raymond, 1997; Raymond et al., 1992) or during entry into WM (Di Lollo et al., 2005; Chun \& Potter, 1995), leaving the second target vulnerable to decay (Chun \& Potter, 1995) or overwriting (Shapiro et al., 1997). Although WM is at the heart of many accounts of the $\mathrm{AB}$, remarkably there is as yet no direct evidence of the involvement of specific brain areas known to be active in WM tasks. The goal of the present report is to redress this omission.

The importance of the present report is further underscored by the fact that not all theories of the $\mathrm{AB}$ posit a vital role for WM (cf. Dux \& Marois, 2009). One example is the account by Di Lollo et al. (2005), the temporary loss of control (TLC) theory. The TLC account suggests that the $\mathrm{AB}$ arises when an attentional filter established to detect T1 is disrupted by the T1 mask. During reconfiguration of the filter stimuli are processed less efficiently giving rise to the $\mathrm{AB}$. If brain areas known to be active in a WM task are shown to play a role in the $\mathrm{AB}$, as the present report demonstrates, then theoretical accounts of this phenomenon not incorporating the interaction of attention and WM will need to be amended.

Outside the AB literature, several previous studies have demonstrated that attention and WM share a strong link. For example, a variety of selective attention theories have proposed that maintaining representations in WM can act to direct attention (e.g., Desimone \& Duncan, 1995; Bundesen, 1990). Furthermore, evidence of a common shared resource exists in the behavioral (e.g., Mayer, Bittner, 
Linden, \& Nikolić, 2007) and functional imaging (Mayer, Bittner, Nikolic, et al., 2007) literatures where processes involved in both selective attention and memory encoding have been shown to interact. Downing (2000) suggested that WM could bias attention toward information consistent with its content (see also Soto, Heike, Humphreys, \& Blanco, 2005; Pratt \& Hommel, 2003). Previous imaging studies have indicated that the cortical site for the attention and memory encoding bottleneck lies in parietal areas (Linden et al., 2003). However, as yet, no functional imaging study has examined the effect of a concurrent WM operation on the cortical structures recruited in the AB task.

Several behavioral investigations have examined the link between WM and the AB (Akyürek, Hommel, \& Jolicour, 2007; Akyürek \& Hommel, 2005, 2006). In the Akyürek and Hommel studies, on each trial, participants were asked to memorize items from a WM array that were probed at the end of the ensuing AB task. WM load and the content relationship between the WM items and the $\mathrm{AB}$ were manipulated by loading WM either with the same content (e.g., letters) as the RSVP distractors, the targets, or neither. Relevant to the present study, the results revealed no specific effect (i.e., interaction) of WM load on the AB in any of the content-type manipulations; overall accuracy was found to decrease as a function of load, but this was no greater in the $\mathrm{AB}$ sensitive $(\sim 100-500 \mathrm{msec})$ interval than in longer intervals where no $\mathrm{AB}$ occurs and attention is fully available.

Importantly, however, the $\mathrm{AB}$ has been determined to interact with WM load when the $\mathrm{AB}$ task requires the use of information stored in WM. Akyürek et al. (2007) changed the WM task to a more active requirement; as before, the task required maintenance of a set of items from a WM array before the start of the $\mathrm{AB}$ task. However, here the T1 task was to determine whether a specified item in the RSVP stream was one of the items from the WM array. The second target (T2) task was the canonical AB task of determining the presence of the letter " $\mathrm{X}$ ". As the number of items in the WM array-and therefore the number of potential matches to $\mathrm{T} 1$ increased-the larger was the $\mathrm{AB}$ magnitude.

Akyürek et al.'s (2007) findings are consistent with reports in the WM literature that have examined the role of attention in WM and have provided evidence of a distinction between maintaining items in WM and acting upon them. For example, Garavan (1997) employed a paradigm where participants were required to keep track of the running total of two types of geometric figures (squares and triangles). Garavan compared the time it took to update the running totals for the geometric shapes when consecutive presentations were either of the same type of shape or were of a different shape. The results showed there was a greater delay when the update required switching the target running total (e.g., updating the square total following a previous updating of the triangle total) compared with updating the same running total a second time (e.g., triangle update following a triangle update). Using a similar approach Oberauer (2002) demonstrated that maintaining two active sets of items in WM, that is, two sets of items on which operations are currently performed, led to a greater decrease in performance than maintaining the same number of items split between an active and a passive set, where the latter required only that the items be maintained.

These results suggest that maintaining items in WM, such as the running totals of the shape types in the Garavan task, can be performed relatively easily, but holding items at a level that allows them to be operated on requires additional resources. The above findings fit neatly into the embedded processes model of WM (Cowan, 1995, 1999). In this view, the availability of an item in WM is dependent on its level of activation, which in turn is dependent on the momentary requirement for its use. The higher state of activation, which is required for the utilization of the contents of WM for cognitive operations, is the "focus of attention" and draws from a capacity limited resource (Cowan, 1995, 1999, 2011; Oberauer, 2002; McElree \& Dosher, 1989). Although there is some debate as to how many items can be contained at the focus of attention (Cowan, 2011), a single item (e.g., McElree \& Dosher, 1989) or several (e.g., Cowan, 1995), there is consensus that raising the activation level of items to bring them into the focus of attention is capacity limited. To apply these findings to the current perspective, the increased $\mathrm{AB}$ on high WM load trials in the Akyürek et al. (2007) study can be interpreted as the result of increasing attentional demands, which arise from maintaining the array of "to be matched" items as the focus of attention, leaving fewer resources available for processing the second target.

Using an electrophysiological approach to address the same question, a recent ERP study employed an AB task to examine the N2pc component elicited by the second target that was significant for the interaction of WM load and T1-T2 interval (Akyürek, Leszczyński, \& Schubö, 2010). The N2pc component is commonly used as a marker of visual attention at an early stage of processing. In the Akyürek et al. study, the N2pc component was maximally suppressed at high WM load and short T1T2 interval, providing compelling evidence that WM operations restrict access to a capacity-limited store shared with the attentional processes required by the $\mathrm{AB}$ task. This result is consistent with electrophysiological research in the WM literature that has shown that the $\mathrm{N} 2 \mathrm{pc}$ component is sensitive to shifts of attention to items held in WM as they become the focus of attention (KuO, Rao, Lepsien, \& Nobre, 2009; see also Eimer \& Kiss, 2010, for a discussion of the N2pc). The results are indicative of a similar mechanism involved in the modulation of attention in perceptual and WM processes to bring about shifts of attention to relevant targets. The present study adds to the understanding of the interaction between attention and WM in the AB task by using functional imaging in combination with a variant of the original Akyürek et al. (2007) paradigm. 
Functional imaging experiments of the $\mathrm{AB}$ have revealed differential modulation of the fronto-parietal attentional network and object processing areas, depending on the presence or absence of an $\mathrm{AB}$ (Johnston, Shapiro, Vogels, \& Roberts, 2007; Shapiro, Johnston, Roberts, \& Zaman, 2007; Kranczioch, Debener, Schwarzbach, Goebel, \& Engel, 2005; Marois, Yi, \& Chun, 2004). For example, Marois et al. (2004) concluded that parietal and lateral frontal areas are recruited to a greater degree on trials where the second target can be reported (i.e., $\mathrm{AB}$ absent) compared with trials when it cannot (i.e., $\mathrm{AB}$ present). The same outcome is observed in the object processing areas of inferotemporal cortex, although the direction of the effect appears to be task specific (cf., Johnston et al., 2007; Shapiro et al., 2007). Similarly, magneto-encephalography has shown that $\mathrm{AB}$ performance is predicted by specific patterns of timelocked signals in the same fronto-parietal-temporal network observed in functional imaging studies (Gross et al., 2004). Given the considerable body of research establishing the cortical sites that form the WM "network" (Linden, 2007; Mohr, Goebel, \& Linden, 2006; D’Esposito, Postle, Ballard, \& Lease, 1999; Owen et al., 1999), it is important to determine how this network functions when attention and WM are required to interact. Specifically, we wish to elucidate the role of WM in the AB task.

We hypothesized that successful detection of T2 would be supported by additional recruitment of the frontoparietal attention network over and above its involvement in the WM task. Thus, AB absent trials should show higher $\mathrm{BOLD}$ activation than $\mathrm{AB}$ present trials. However, we predicted this pattern of activity only under low WM load, because a high WM load in combination with an attentional demand has been shown to reveal a pattern of underadditivity (Mayer, Bittner, Nikolic, et al., 2007). On the basis of the findings by Johnston et al. (2007) and Shapiro et al. (2007), which have shown BOLD activity associated with $\mathrm{AB}$ present versus $\mathrm{AB}$ absent trials to reverse as the attentional network is placed under high load, we predicted a reversed pattern of activity to be shown under conditions of high WM load.

\section{METHODS}

\section{Participants}

Sixteen participants (nine women and seven men) with a mean age of $22.5 \pm 1.4$ years were recruited from the local community. All participants were right-handed with no history of neurological disorders and provided informed consent before the start of the study. All procedures were approved by the ethics board of Bangor University.

\section{Design}

A $2 \times 2$ repeated measures factorial design was implemented with factors of WM Load (one or three items) and $\mathrm{AB}$ lag (T1-T2 interval; short or long). Because the contrasts of most interest are those at the short lag, where we typically find a mixture of both $\mathrm{AB}$ present (WM task successful, "X" not detected) and AB absent trials (WM task successful, "X" detected), the proportion of trials at the short lag to the long lag was approximately $2: 1$. The behavioral-dependent measure was T2 accuracy, conditional on a correct T1 judgment.

\section{Stimuli}

Ten stimuli were used for the T1 and WM items, five items designated "target," to-be-remembered items and five "nontargets" that the participant was to ignore. The five target items were a set of complete edge (i.e., no line break), closed contour geometric shapes (circle, triangle, square, hexagon, and star). The five nontarget items were identical to the five target items, save their boundary edges were incomplete (i.e., a dashed "closed" contour; see Figure 1). The stimuli subtended approximately $4^{\circ}$ of visual angle across their widest dimension. Selection of the stimuli for use in the WM array, on each trial, was random with no replacement. On Load 1 trials, the WM array consisted of one target item and two nontarget items; for Load 3 trials, three target items only were selected. WM array items were displayed in the middle of the screen in a line across the display vertical midline, with the position of the target item in array, in the Load 1 condition, randomly selected on each trial. On WM match trials, the T1 item was randomly selected from one of the target items used in the preceding WM array; for WM nonmatch trials, the T1 item was selected randomly from the stimuli that were not used in the WM array. The T1 mask, which followed T1 in the RSVP, was created by overlaying all five shapes to make a composite stimulus. The T2 and distractor items in the RSVP consisted of upper case alphabetic characters (save S, O, Z, I, and B, which were excluded to avoid confusion with visually similar numeric characters). On target present trials, T2 was an "X," on nonpresent trials it could be any other valid character. T2 was present on two thirds of the trials; the remaining third were nontarget trials. The RSVP stream consisted of 16 items, including T1 and T2, each appearing for $32 \mathrm{msec}$ with a $16 \mathrm{msec}$ ISI. T2 could appear at one of two lags, either at the short lag (192 msec post-T1) or long lag (688 msec post-T1). The experiment consisted of seven functional imaging runs, each consisting of 26 trials (16 at the short lag, 10 at the long lag), and in each case, half of those trials were at a WM load of 1 ( 8 trials at the short lag, 5 trials at the long lag), and half were at a WM load of 3 (8 trials at the short lag, 5 trials at the long lag). A schematic of the trial structure is shown in Figure 1.

\section{Apparatus}

Stimuli were back-projected from a Taxan U6 projector (Kaga Components Co., Ltd., Tokyo, Japan) running at $60 \mathrm{~Hz}$ with a resolution of $1024 \times 768$ pixels onto a screen located at the head end of the MRI scanner. The presented 
Figure 1. Schematic of the $\mathrm{AB}-\mathrm{WM}$ trial procedure.

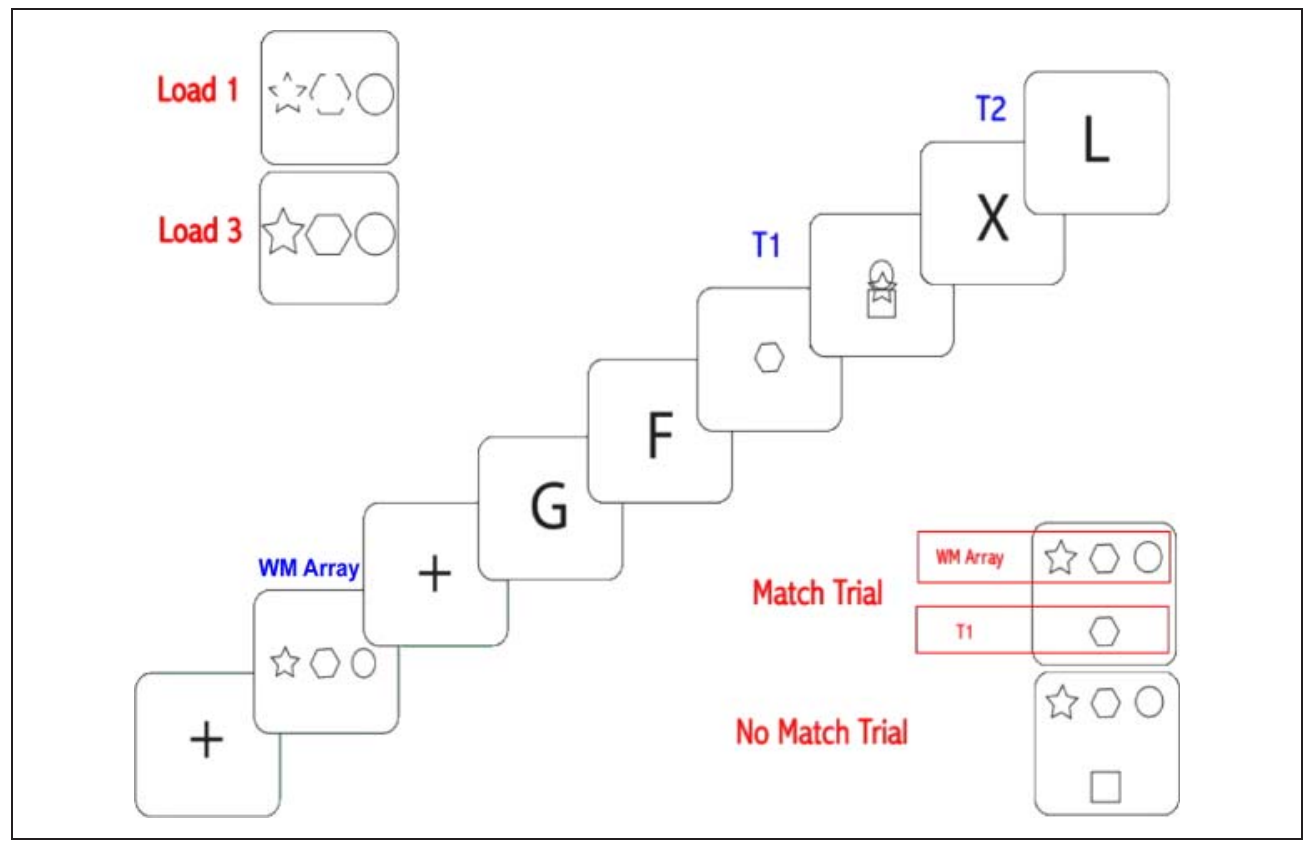

images were viewed by the participants via a mirror mounted on the head coil. Responses were collected via a fiber-optic button box (Current Designs, Inc., Philadelphia, PA). The experiment was run from a laptop using the psychology presentation software, E-Prime (Psychology Software Tools, Inc., Pittsburgh, PA).

\section{Procedure}

Each trial began with a fixation cross, which remained on-screen until triggered by the MRI scanner. Trials were spaced 14 sec apart to allow, along with a randomized presentation of trial type, efficient estimation of the BOLD response for each trial type. At the beginning of each trial, the fixation cross was replaced with a centrally presented WM array containing three items; depending on the trial type, the display could either contain three complete geometric shapes (Load 3) or one complete geometric shape and two further geometric shapes but with incomplete edges (Load 1). Participants were told to ignore any shapes that were "incomplete" (see Figure 1). The T1 target, to which the contents of the WM array were matched, was never one of the "incomplete" shapes, and it was always present and only ever a full, complete geometric shape. The WM array stayed on-screen for $3 \mathrm{sec}$. There was a 1-sec fixation screen after the WM array was removed before the start of the RSVP stream. The two targets, T1 and T2, were embedded within the RSVP stream. The first target T1 and its mask were the only geometric shapes in what was otherwise a stream of letters. The participant's first task was to match the geometric shape in the stream (T1) to the items presented in the WM array; participants were told not to respond immediately but await an instruction at the end of each trial. The participant's second task was to report whether they saw the "X" in the RSVP. As with the T1 task, T2 report was withheld until the end of the trial. At the completion of the RSVP, the participants were prompted for their responses in the order of appearance, that is, T1 then T2. The participants gave their T1 response upon seeing the prompt, "Match"; similarly, the T2 response was provided in response to an "X?" prompt. Both prompts were presented centrally.

\section{Imaging Parameters}

BOLD-sensitive images were collected on a Philips Achieva 3-T MRI Scanner (Philips, Best, the Netherlands) using a SENSE head coil. A total of 260 imaging volumes (TR = $2000 \mathrm{msec}, \mathrm{TE}=35 \mathrm{msec}$, in-plane resolution $=3 \mathrm{~mm} \times$ $3 \mathrm{~mm}$, flip angle $=90^{\circ}, 3.5-\mathrm{mm}$ thick with no gap) were collected each run; images were collected in an axial orientation and covered the whole cerebrum.

\section{Image Processing}

All preprocessing and analyses were conducted using BrainVoyager QX (Brain Innovations, B.V., Maastricht, the Netherlands). For each run, all the images were coregistered to the first image of the first functional run (functional template) to correct for intra-run and inter-run participant movement, such that all the collected images were in the same space. The functional template was then coregistered with a high-resolution 3-D T1-weighted anatomical image that was collected at the end of the experiment. Once the T1-weighted image had been transformed into the Talairach stereotaxic space (Talairach \& Tournoux, 1988), from the known transformation matrix of the T1-weighted image to Talairach space, the functional 
images were also transformed into Talairach space before being analyzed. All functional volumes were spatially smoothed using an 8-mm 3-D Gaussian kernel and filtered, low and high pass, to remove biological artifacts and long-term drift. The data were analyzed using the general linear model approach. Regressors were created corresponding to the following trial types: short lag $\mathrm{AB}$ present Load 1 ( $\mathrm{T} 1$ correct, $\mathrm{T} 2$ incorrect), short lag $\mathrm{AB}$ absent Load 1 (T1 correct, T2 correct), short lag AB present Load 3 (T1 correct, T2 incorrect), short lag AB absent Load 3 (T1 correct, T2 correct), trials of no interest (long lag trials and trials where T1 was incorrectly matched). Each regressor was created to cover the period between WM array onset and the end of the RSVP stream; the total length of this regressor, therefore, covered a period of $4.8 \mathrm{sec}$. Each regressor was convolved with a hemodynamic response function (Friston et al., 1998) that models the delay and spread of the measured BOLD response to the neural event. Where whole-brain analyses are conducted, data were analyzed using the random effects approach with the threshold for acceptance set at $p<.05$ corrected for multiple comparisons using the cluster correction statistical threshold correction, for which an alpha value of $p<$ .05 was used. The cluster threshold approach controls the likelihood of Type I error by determining the distribution of cluster size given the number of supra-threshold voxels in the image.

\section{RESULTS}

\section{Behavioral Data}

The behavioral data from the T1 task were entered into a 2 (WM Load: 1 or 3) $\times 2$ (Lag: short or long) repeated measures ANOVA. The results showed only a main effect of WM Load $(F(1,15)=19.4, p<.05)$. Examination of the means reveals matching T1 to three items (Load 3) yields a lower mean accuracy $(86.8 \pm 13.6 \%)$ than matching $\mathrm{T} 1$ to a single item (Load 1; $93.5 \pm 11.5 \%$ ).
Analysis of the T2 data, conditional on correct $\mathrm{T} 1,{ }^{2}$ using a repeated measures ANOVA with the same factors and levels as for T1, reveals a main effect of WM Load $(F(1$, $15)=20.7, p<.05)$ and $\operatorname{Lag}(F(1,15)=61.9, p<.05)$. Critically a significant interaction was found between WM Load and Lag, showing that increasing WM load reduces correct detection of T2 $(F(1,15)=11.0, p<.05)$ but only at the short lag. Figure 2 shows the means for each condition. Follow-up simple contrasts show significantly poorer T2 detection for Load 3 relative to Load 1 at the short lag $(t(15)=5.2, p<.05 ;$ Load $3: 40.6 \% \pm 18.9 \%$, Load $1: 57.3 \% \pm$ $23.3 \%)$ but not at the long lag $(t(15)=.61, n s$; Load $3: 85.8 \% \pm$ 11.6, Load 1: $85.6 \% \pm 12.1)$. The behavioral data, thus, reveal a more pronounced $\mathrm{AB}$ under high WM load. The lack of a significant difference in the false alarm rate for WM Loads 1 and 3 at the short lag removes the concern that "guessing" can account for this outcome.

\section{Functional Imaging Results}

\section{Areas Sensitive to WM Load}

To determine brain areas involved in the WM component of the task a contrast was computed between the high versus the low WM load conditions, irrespective of $\mathrm{AB}$ present/absent outcome. Several areas were seen to be active (see Table 1 for a full list), in particular, significant load-dependent activation was found in bilateral inferior and superior parietal cortex, bilateral inferotemporal cortex, and several regions in PFC. These areas have been reported previously in many imaging studies of WM (see Linden, 2007, for a review) and, relevant to the issue at hand, include regions that have been implicated in $\mathrm{AB}$ tasks (e.g., Shapiro et al., 2007; Kranczioch et al., 2005; Marois et al., 2004). Apart from two regions, the response pattern shown in Table 1 is due to a higher level of activation in the high load condition compared with the low load condition. The only exceptions are the activations seen in the anterior cingulate gyrus (Talairach coordinate: 2, 44, 13) and a region in the left middle temporal gyrus (Talairach
Figure 2. Behavioral results of the AB-WM experiment, shown are overall T1 accuracy as a function of WM load (left) and $\mathrm{T} 2$ accuracy, conditional on correct T1, as a function of WM load (right). Each mean is shown with its associated $S E$.

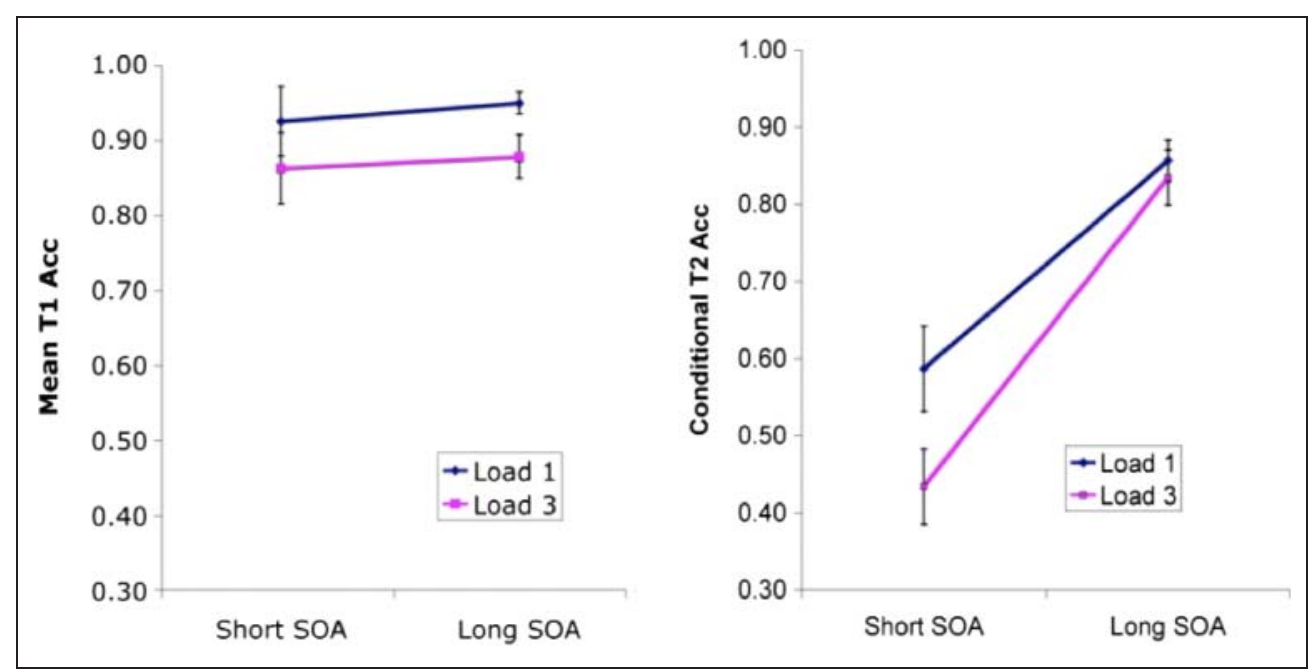


Table 1. Regions Significantly Active $(p<.05, p<.05$ Cluster Corrected) for the High versus Low WM Load Contrast

\begin{tabular}{|c|c|c|c|c|}
\hline Region & Hemisphere & Brodmann's Area & Talairach Coordinate $(x, y, z)$ & Max $t$ Value \\
\hline \multirow[t]{2}{*}{ Lingual gyrus } & $\mathrm{R}$ & 18 & $12-75-10$ & 5.91 \\
\hline & $\mathrm{L}$ & & $-8-73-5$ & 5.16 \\
\hline Fusiform gyrus & $\mathrm{L}$ & 37 & $-39-49-21$ & 4.07 \\
\hline Middle temporal gyrus & $\mathrm{L}$ & 39 & $-51-7018$ & -3.60 \\
\hline Superior temporal gyrus & $\mathrm{R}$ & 39 & $32-5518$ & 5.18 \\
\hline \multirow[t]{2}{*}{ SPL/IPL } & $\mathrm{L}$ & $7 / 39$ & $-24-6527$ & 6.62 \\
\hline & $\mathrm{L}$ & $7 / 40$ & $-31-5226$ & 6.03 \\
\hline SPL & $\mathrm{R}$ & 7 & $26-6631$ & 4.86 \\
\hline IPL & $\mathrm{L}$ & 40 & $-35-4537$ & 4.19 \\
\hline Precentral gyrus & $\mathrm{L}$ & 4 & $-43-1544$ & 7.30 \\
\hline \multirow[t]{2}{*}{ MFG/precentral gyrus } & $\mathrm{L}$ & 6 & -41536 & 4.66 \\
\hline & $\mathrm{R}$ & 6 & 48646 & 5.10 \\
\hline Inferior frontal gyus & $\mathrm{L}$ & 44 & -46821 & 4.38 \\
\hline Superior frontal gyrus & $\mathrm{L}$ & 6 & $-7-954$ & 6.83 \\
\hline MFG & $\mathrm{R}$ & 8 & 313146 & 3.47 \\
\hline Superior frontal gyrus & $\mathrm{L}$ & 9 & -305039 & 4.90 \\
\hline SPL & $\mathrm{R}$ & 7 & $31-4356$ & 4.17 \\
\hline Medial frontal gyrus/cingulate gyrus & $\mathrm{R}$ & 9 & 24413 & -5.28 \\
\hline
\end{tabular}

$\mathrm{R}=$ right; $\mathrm{L}=$ left.

coordinate: $-51,-70,18)$, where an increase in WM load leads to a relative deactivation, a finding that is consistent with reports of activation decreases in the "default mode network" to increasing cognitive demand (e.g., Mayer, Roebroeck, Maurer, \& Linden, 2010). Overall, with the exception of the above two regions, the pattern of higher activity in a predominantly fronto-parietal network at high WM load is consistent with previous studies (cf. Linden, 2007).

\section{Areas Sensitive to the $A B$}

A contrast examining the effect of $\mathrm{AB}$ ( $\mathrm{AB}$ present vs. $\mathrm{AB}$ absent) was performed to determine which areas were more active as a function of successful deployment of attention to the second target. Several sites were identified in this contrast (see Table 2) that fall within the traditional bounds of the fronto-parietal attention network, in line with previous findings of non-ROI imaging studies examining the $\mathrm{AB}$ (e.g., Kranczioch et al., 2005; Marcantoni, Lepage, Beaudoin, Bourgouin, \& Richer, 2003). In particular, activation was revealed in inferior and superior parietal cortices and the inferior frontal gyrus, in addition to sites in the "ventral" object-processing stream of occipito-temporal regions. Importantly, for almost all of the sites labeled in Table 2, there was more activation when the second target could be successfully detected, which conforms with previous studies of AB (e.g., Kranczioch et al., 2005; Marois et al., 2004). The exception occurs in the inferior occipital/fusiform gyrus region (Talairach coordinate: $-38,-82,-8$ ) and a region in inferior temporal cortex (Talairach coordinate: -52 , $-63,-14)$ that is often referred to as the lateral occipital complex, which has been shown to be involved in object processing. Enhanced activation associated with trials where the second target is undetected (i.e., an AB occurs) has been reported previously for these areas and attributed to an attempt to process the second target in the face of insufficient attentional resources. (Johnston et al., 2007; Shapiro et al., 2007; Kranczioch et al., 2005).

\section{Areas Sensitive to the Interaction between WM Load and $A B$}

Finally, areas that showed an interaction between WM load and $\mathrm{AB}$ were examined using a contrast of [High WM load (AB present $-\mathrm{AB}$ absent) - Low WM load ( $\mathrm{AB}$ present $\mathrm{AB}$ absent)]. Brain areas that met this criterion are located in prefrontal, parietal, and inferotemporal regions (see Table 3 for a full list); many of these regions yield statistical significance in the main effect contrasts of WM load and AB. Although there is overlap among the main effects and interaction contrasts, there are areas active in the interaction contrast that are unique. In particular, the interaction contrast reveals more widespread activation in the ventral brain 
Table 2. Regions Significantly Active $(p<.05, p<.05$ Cluster Corrected $)$ for the AB versus No-AB Contrast

\begin{tabular}{|c|c|c|c|c|}
\hline Region & Hemisphere & Brodmann's Area & Talairach Coordinate $(x, y, z)$ & Max $t$ Value \\
\hline Inferior temporal cortex & $\mathrm{L}$ & 37 & $-52-63-14$ & 3.01 \\
\hline Inferior occipital gyrus/fusiform gyrus & $\mathrm{L}$ & $19 / 18$ & $-38-82-8$ & 2.72 \\
\hline \multirow[t]{2}{*}{ Inferior frontal gyrus } & $\mathrm{L}$ & 46 & -384213 & -3.68 \\
\hline & $\mathrm{L}$ & $9 / 44$ & -541525 & -2.91 \\
\hline Superior temporal gyrus & $\mathrm{L}$ & 22 & $-43-5619$ & -2.52 \\
\hline IPL & $\mathrm{L}$ & 40 & $-48-6040$ & -3.91 \\
\hline SPL & $\mathrm{R}$ & $7 / 40$ & $30-3953$ & -4.65 \\
\hline \multirow[t]{2}{*}{ Precentral gyrus } & $\mathrm{L}$ & 4 & $-20-1865$ & -5.58 \\
\hline & $\mathrm{L}$ & 4 & $-37-756$ & -4.69 \\
\hline
\end{tabular}

$\mathrm{R}=$ right; $\mathrm{L}=$ left.

areas, for example, superior temporal gyrus (Talairach coordinate: $51,-54,21$ ), middle temporal gyrus (Talairach coordinate: $49,-39,5$ ), and fusiform gyrus (Talairach coordinate: $29,-74,-2)$ consistent with the requirement that the $\mathrm{AB}$ task requires object processing. Two sites seen in this interaction contrast are of particular interest because they overlap with clusters found in our main effects. One of these areas is a cluster in middle frontal gyrus (MFG; Talairach coordinate: $-43,44,20)$, which overlaps with an area found in the $\mathrm{AB}$ present vs. absent contrast (Talairach

Table 3. Regions Significantly Active $(p<.05, p<.05$ Cluster Corrected) for the WM Load $\times$ AB Interaction

\begin{tabular}{|c|c|c|c|c|}
\hline Region & Hemisphere & Brodmann's Area & Talairach Coordinate $(x, y, z)$ & Max $t$ Value \\
\hline Precentral gyrus & $\mathrm{R}$ & 4 & $50,1,43$ & 3.47 \\
\hline \multirow[t]{2}{*}{ Postcentral gyrus } & $\mathrm{L}$ & $3 / 5$ & $-42-1643$ & 3.19 \\
\hline & $\mathrm{R}$ & 1 & $30-2856$ & 4.33 \\
\hline \multirow[t]{2}{*}{ Superior frontal gyrus } & $\mathrm{R}$ & 8 & 262555 & 3.42 \\
\hline & $\mathrm{L}$ & 6 & $-21-1064$ & 2.72 \\
\hline Medial frontal gyrus & $\mathrm{L}$ & 6 & $-6-1356$ & 3.59 \\
\hline \multirow[t]{3}{*}{ MFG } & $\mathrm{R}$ & $8 / 9$ & 332749 & 4.06 \\
\hline & $\mathrm{R}$ & 6 & 49046 & 3.47 \\
\hline & $\mathrm{L}$ & 46 & -434420 & 3.02 \\
\hline Inferior frontal gyrus & $\mathrm{R}$ & 45 & 60418 & 3.01 \\
\hline SPL & $\mathrm{R}$ & 7 & $27-6421$ & 3.89 \\
\hline \multirow[t]{2}{*}{ IPL } & $\mathrm{L}$ & 40 & $-36-5028$ & 2.68 \\
\hline & $\mathrm{R}$ & & $33-2946$ & 3.77 \\
\hline Fusiform gyrus/inferior temporal cortex & $\mathrm{R}$ & 18 & $29-74-2$ & 3.30 \\
\hline \multirow[t]{2}{*}{ Superior temporal gyrus } & $\mathrm{R}$ & 22 & $498-7$ & 3.08 \\
\hline & $\mathrm{R}$ & 22 & $51-5421$ & 2.66 \\
\hline Middle temporal gyrus & $\mathrm{R}$ & 22 & $49-395$ & 2.72 \\
\hline Inferior frontal gyrus & $\mathrm{R}$ & 47 & 48327 & 3.42 \\
\hline \multirow[t]{2}{*}{ Middle occipital gyrus/occipito-parietal } & $\mathrm{R}$ & 19 & $33-7319$ & 3.84 \\
\hline & $\mathrm{L}$ & & $-28-7420$ & 3.08 \\
\hline Cuneus & $\mathrm{R}$ & 18 & $9-8023$ & 2.64 \\
\hline
\end{tabular}

$\mathrm{R}=$ right $; \mathrm{L}=$ left. 


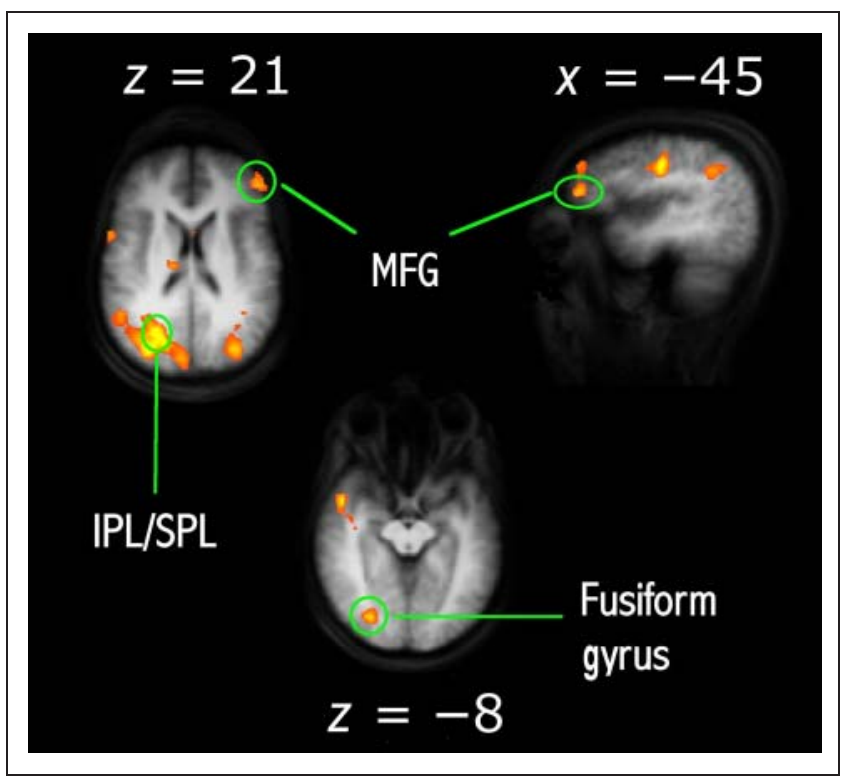

Figure 3. Areas active in the functional imaging data in the $A B \times$ WM Load interaction shown overlaid on the average of all participant's anatomical images. Areas shown are the clusters located in the SPL/IPL boundary and MFG and a cluster in the fusiform gyrus.

coordinate: $-38,42,13)$. The second site is a cluster of activity bordering the inferior parietal lobe (IPL) and superior parietal lobe (SPL; Talairach coordinate: $27,-64,21$ ), which corresponds to activity revealed in the WM contrast of high vs. low WM load (Talairach coordinate: 26, -66, 31). Figure 3 displays several key sites that are significant in the interaction contrast, whereas Figure 4 shows the mean beta values for four of the significant clusters representative of the response observed in all regions. For all regions identified via this contrast, we observed that the neural response at low WM loads was greater when T2 could be detected, that is, no AB occurred. This pattern was reversed for high WM loads, where activity in these same regions is greater when T2 could not be detected, that is, an $\mathrm{AB}$ occurred.

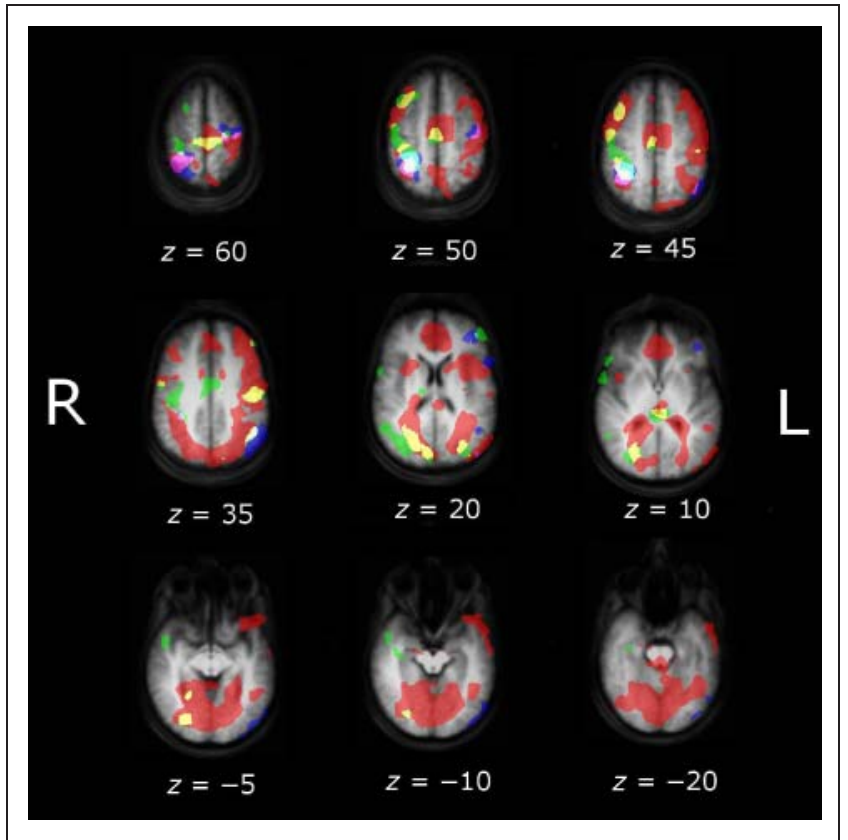

Figure 5. Nine axial slices show the areas responsive to the contrast of WM Load (red), AB (blue), and the interaction of WM Load $\times A B$ (green). Overlap between areas active in each condition is denoted through color additions according to the red-green-blue system (e.g., yellow: overlap between WM Load [red] and WM Load $\times$ Interaction [green]).

Figure 5 shows the areas of significant activity found for all the above contrasts overlaid on the same anatomical image.

\section{DISCUSSION}

The purpose of the present experiment was to understand the neural locus of the interaction between WM and attention in the context of the $\mathrm{AB}$. In addition to the vital role of attention in the AB outcome (cf. Raymond et al., 1992), most theories of the $\mathrm{AB}$ specify a prominent role for WM in both target tasks, either at encoding (e.g., Chun \& Potter,
Figure 4. A graph of the mean beta values in examplar regions, fusiform gyrus (FG; $29-74$ -2), MFG (-43 44 20), middle temporal gyrus (MTG; $49-39$ 5), and SPL/IPL (27 -64 21).

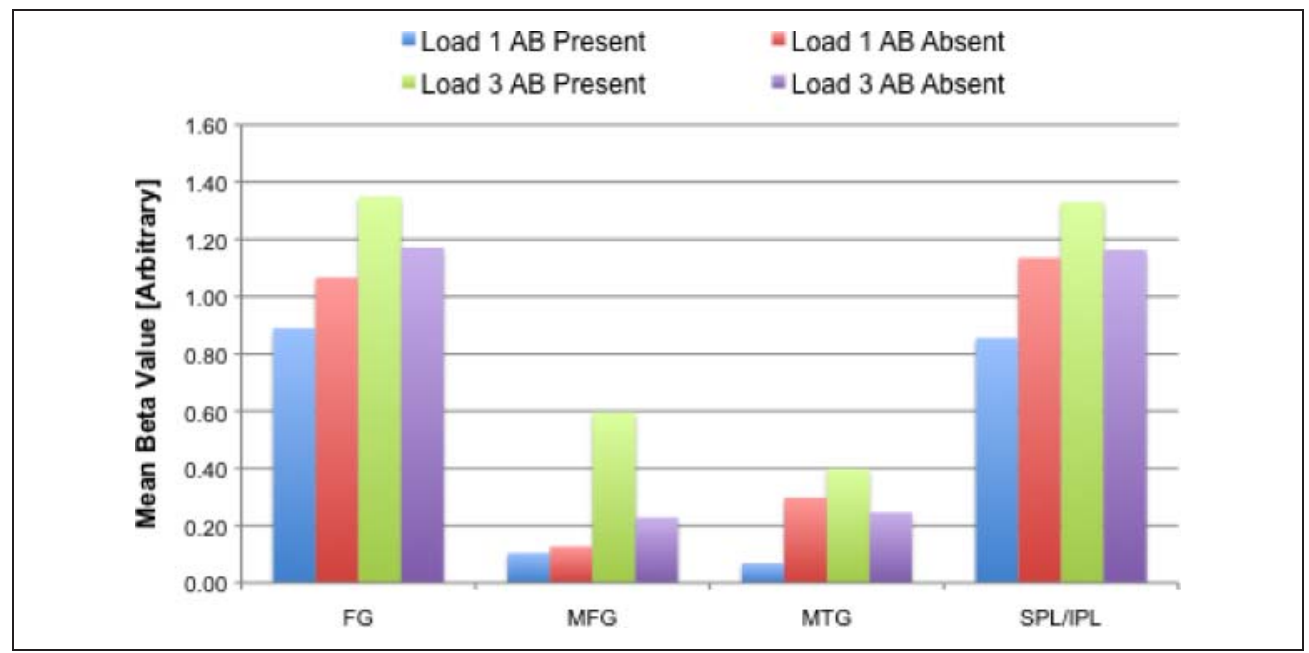


1995) or retrieval (e.g., Shapiro et al., 1994). Although this interaction has been revealed using behavior and electrophysiology approaches, the neuroanatomical locus of this interaction has yet to be elucidated.

To identify the key cortical regions involved in the interaction of these two cognitive phenomena, we employed a delayed matching-to-sample task (cf. Sternberg, 1966) as used by Akyürek et al. (2007). The behavioral results of the present study replicate those of Akyürek et al., despite both methodological and stimulus differences, and confirm the expected interaction; participants were significantly worse at detecting the second target in an $\mathrm{AB}$ task when they had to match the T1 target to a WM array of three items compared with matching a WM array of only one item. Importantly, this reduction in performance occurred only at the short "AB-sensitive" lag, supporting the previous finding that WM and attention interact in the $\mathrm{AB}$ task. Our motivation for the present study was to determine the cortical regions exhibiting an analogous interaction to the behavioral result and to compare these with areas known to be active in both WM and $\mathrm{AB}$ tasks.

It is important to point out that, for those areas revealing an interaction between WM and $\mathrm{AB}$, the pattern of activity cannot be because of a difference in WM maintenance per se; in both cases, the contrast of $\mathrm{AB}$ present versus $\mathrm{AB}$ absent is made within the same WM load condition and only on trials where the T1 task is correct. Similarly, sites involved solely in the maintenance of the stimuli are unlikely to be revealed by this interaction, given that attempts to find an interaction with WM maintenance (i.e., in a task not requiring WM to perform the $\mathrm{T} 1$ task) and the $\mathrm{AB}$ have failed to reveal such an effect (Akyürek \& Hommel, 2005, 2006).

The contrasts of high versus low WM load and $\mathrm{AB}$ present versus $\mathrm{AB}$ absent revealed a number of cortical sites that have been reported previously in WM and attention-based tasks (e.g., Johnston et al., 2007; Linden, 2007; Shapiro et al., 2007; Bledowski et al., 2006; Kranczioch et al., 2005; Marois et al., 2004; Marcantoni et al., 2003; D’Esposito et al., 1999). In both contrasts, significant activity was seen in parietal cortex, various prefrontal and frontal areas, and object-processing sites in the inferotemporal cortex. However, as the present study was not designed specifically to contrast these particular trial types and importantly any such differences are affected by the interaction between our attention and WM manipulations, we will refrain from overinterpreting these results. The difficulty in interpreting the main effects of covarying WM and attention has recently been highlighted by Mayer, Bittner, Nikolic, et al. (2007), who have shown changes in cortical response during a WM task as a function of attentional load.

\section{Mechanisms of Attention under WM Load}

The primary purpose of the present investigation is to further our understanding of the role played by WM in the $\mathrm{AB}$. The importance of this goal is underscored by the prominent position played by the construct of WM in many accounts of the $\mathrm{AB}$ but which has not yet been verified by direct examination of brain activity as is revealed by functional imaging. The key finding is that we observed increased activity on $\mathrm{AB}$ absent (compared with $\mathrm{AB}$ present) trials at low WM loads but a reversal in activity at high WM loads. The former outcome is consistent with previous findings, which suggest that these brain regions are active during the deployment of attention to visual events (Marois et al., 2004; Coull \& Nobre, 1998). This outcome is also consistent with the notion that conscious awareness of a stimulus is registered in a fronto-parietal network, as suggested by Dehaene's "global workspace" account (Dehaene, Changeux, Naccache, Sackur, \& Sergent, 2006). Using magneto-encephalography, a similar network was identified by Gross et al. (2004) with synchronisation between frontal and parietal regions being predictive of successful first and second target performance in an $\mathrm{AB}$ task.

The latter pattern, that of increased activity for $\mathrm{AB}$ present relative to absent trials at high WM load, is consistent with findings previously reported (Johnston et al., 2007; Shapiro et al., 2007; Kranczioch et al., 2005). In these studies, the larger BOLD response on $\mathrm{AB}$ present trials was attributed to increased neural activity occurring when participants attempted to identify targets under cognitively adverse conditions. Such an account, on the basis of the notion of increased depletion of shared resources, is consistent with the finding from a recent ERP study of the interaction of WM and $A B$ (Akyürek et al., 2010). In this study, a similar combination of high WM load and a short lag led to suppression of the N2pc waveform attributed to the second target, a waveform that acts as a marker of the deployment of attentional resources for visual processing. This would be consistent with the notion that the $\mathrm{N} 2 \mathrm{pc}$ is sensitive to internal shifts of attention to items held in WM (Kuo et al., 2009). At high WM loads, the reduction in the N2pc may reflect the failure to shift attention successfully to the second target because resources are being utilized by items in the WM as they are made the focus of attention such that T1 can be successfully matched. Taken as a whole, these findings suggest that reduced attentional resources arising from the $\mathrm{AB}$, interacting with high WM demands, leads to a poor representation of the second target, in turn yielding an increased BOLD response as the brain struggles to bring the second target to conscious access. A possible alternative account, which will require additional experimentation to verify, suggests that the interaction of WM and the AB leads to the recruitment of new brain areas to solve the same problem highlighted above.

A question still remains as to how we can reconcile the difference in the response of cortical regions on $\mathrm{AB}$ present versus $\mathrm{AB}$ absent trials at high WM load compared with low WM load, assuming that the nature of the resource depletion is the same. In particular why do the $\mathrm{AB}$ present trials at low load not capitalize on the, apparently, available resources that are implied by the even larger BOLD response produced on $\mathrm{AB}$ absent trials at high WM load? From the current experiment, there is no clear way to 
empirically test how this occurs; however, we can speculate on how this pattern may arise. If we assume a level of shared functional architecture between maintenance and processing of items in WM, the output of cortical regions would be the summation of contributions from both general and capacity limited resources. As shown by Akyürek and Hommel (2005, 2006), maintenance operations do not appear to interact with the $\mathrm{AB}$; however, there does appear to be an overall deficit in performance when maintaining a high load compared with a low load. Assuming a shared architecture, then we may expect the average value of the cortical output, as measured by the BOLD response, to scale with load, with smaller variations being accounted for by availability of resources from the capacity-limited pool. We remain agnostic as to the level of shared functional architecture between maintenance and updating operations given that such a goal was not the focus of this experiment, but an account such as this would explain the present pattern of results.

What then for models of the $\mathrm{AB}$ ? The current results replicate those of Akyürek and his colleagues, strongly suggesting that WM plays a key role in the AB. Importantly, the present report goes further to reveal the pattern of cortical activity associated with the interaction of attention and WM in a number of brain areas previously associated with conscious awareness (Dehaene et al., 2006). This pattern of results is consistent with models that propose limitations in resources at the level of WM as key to the AB outcome (e.g., Bowman \& Wyble, 2007; Chun \& Potter, 1995; Shapiro et al., 1994). Whether the role played by WM operates at the level of encoding or retrieval (or both) cannot be ascertained by the present study as we imaged throughout both of these processes. The present results further suggest that models of the $\mathrm{AB}$ that do not incorporate a specific role for WM may not fully characterize this phenomenon. For example, Di Lollo et al. (2005) proposed in their TLC model that a filter established for the first target takes time to reconfigure for the second target, allowing the second target to fail to be processed. Unless control over the filter is acknowledged to depend on WM constraints, this model cannot account for the present findings.

\section{Conclusion}

In conclusion, the use of a hybrid WM-AB paradigm has successfully identified specific brain regions, notably the MFG and the parietal cortex, which play a role when attention and WM demands interaction to produce the well-known phenomenon of the $\mathrm{AB}$. The present results underscore the need for any viable account of the $\mathrm{AB}$ to incorporate WM as one of its primary tenets.

\section{Acknowledgments}

This research was supported by a project grant (071944) from the Wellcome Trust to Kimron Shapiro.
Reprint requests should be sent to Stephen J. Johnston, School of Social Sciences, Brunel University, Uxbridge, UB8 3PH, UK, or via e-mail: stephen.johnston@brunel.ac.uk.

\section{Notes}

1. We refer to visual working memory as the active manipulation of the contents of temporarily stored items in visual short term, as distinct from "short-term" memory where items are solely retained over a limited period.

2. This conditional treatment of the $\mathrm{T} 2$ data ensures that any changes in $\mathrm{T} 2$ performance as a function of our independent variables are not because of lapses in attention to T1.

\section{REFERENCES}

Akyürek, E. G., \& Hommel, B. (2005). Short-term memory and the attentional blink: Capacity versus content. Memory and Cognition, 33, 654-663.

Akyürek, E. G., \& Hommel, B. (2006). Memory operations in rapid serial visual presentation. European Journal of Cognitive Psychology, 18, 520-536.

Akyürek, E. G., Hommel, B., \& Jolicœur, P. (2007). Direct evidence for a role of working memory in the attentional blink. Memory and Cognition, 35, 621-627.

Akyürek, E. G., Leszczyński, M., \& Schubö, A. (2010). The temporal locus of the interaction between working memory consolidation and the attentional blink. Psychophysiology, 47, 1134-1141.

Bledowski, C., Cohen Kadosh, K., Wibral, M., Rahm, B., Bittner, R. A., Hoechstetter, K., et al. (2006). Mental chronometry of working memory retrieval: A combined functional magnetic resonance imaging and event-related potentials approach. Journal of Neuroscience, 26, 821-829.

Bowman, H., \& Wyble, B. (2007). The simultaneous type, serial token model of temporal attention and working memory. Psychological Review, 114, 38-70.

Bundesen, C. A. (1990). Theory of visual attention. Psychological Review, 97, 523-547.

Chun, M. M., \& Potter, M. C. (1995). A two-stage model for multiple target detection in rapid serial visual presentation. Journal of Experimental Psychology: Human Perception and Performance, 21, 109-127.

Coull, J. T., \& Nobre, A. C. (1998). Where and when to pay attention: The neural systems for directing attention to spatial locations and to time intervals as revealed by both PET and fMRI. Journal of Neuroscience, 18, 7426-7435.

Cowan, N. (1995). Attention and memory: An integrated framework. New York: Oxford University Press.

Cowan, N. (1999). An embedded-processes model of working memory. In A. Miyake \& P. Shah (Eds.), Models of working memory: Mechanisms of active maintenance and executive control (pp. 62-101). Cambridge: Cambridge University Press.

Cowan, N. (2011). The focus of attention as observed in visual working memory tasks: Making sense of competing claims. Neuropsychologia, 49, 1401-1409.

Dehaene, S., Changeux, J. P., Naccache, L., Sackur, J., \& Sergent, C. (2006). Conscious, preconscious, and subliminal processing: A testable taxonomy. Trends in Cognitive Sciences, 10, 204-211.

Desimone, R., \& Duncan, J. (1995). Neural mechanisms of selective visual attention. Annual Review of Neuroscience, 18, 193-222.

D’Esposito, M., Postle, B. R., Ballard, D., \& Lease, J. (1999). Maintenance versus manipulation of information held in 
working memory: An event-related fMRI study. Brain $\mathcal{E}$ Cognition, 41, 66-86.

Di Lollo, V., Kawahara, J.-I., Ghorashi, S. M., \& Enns, J. T. (2005). The attentional blink: Resource depletion or temporary loss of control? Psychological Research, 69, 191-200.

Downing, P. (2000). Interactions between visual working memory and selective attention. Psychological Science, 11, 467-473.

Dux, P. E., \& Marois, R. (2009). The attentional blink: A review of data and theory. Attention, Perception \& Psychophysics, $71,1683-1700$

Eimer, M., \& Kiss, M. (2010). The top-down control of visual selection and how it is linked to the N2pc component. Acta Psychologica, 135, 100-102.

Friston, K. J., Fletcher, P., Josephs, O., Holmes, A., Rugg, M. D., \& Turner, R. (1998). Event-related fMRI: Characterising differential responses. Neuroimage, 7, 30-40.

Garavan, H. (1997). Serial attention within working memory. Memory \& Cognition, 26, 263-276.

Gross, J., Schmitz, F., Schnitzler, A., Kessler, K., Shapiro, K. L., Hommel, B., et al. (2004). Modulation of long-range neural synchrony reflects temporal limitations of visual attention in humans. Proceedings of the National Academy of Sciences, U.S.A., 101, 13050-13055.

Johnston, S. J., Shapiro, K. L., Vogels, W., \& Roberts, N. J. (2007). Imaging the attentional blink: Perceptual versus attentional limitations. NeuroReport, 18, 1475-1478.

Kranczioch, C., Debener, S., Schwarzbach, J., Goebel, R., \& Engel, A. K. (2005). Neural correlates of conscious attention in the attentional blink. Neuroimage, 24, 704-714.

Kuo, B.-C., Rao, A., Lepsien, J., \& Nobre, A. C. (2009). Searching for targets within the spatial layout of visual short-term memory. Journal of Neuroscience, 29, 8032-8038.

Linden, D. E. J. (2007). The working memory networks of the human brain. The Neuroscientist, 13, 257-267.

Linden, D. E. J., Bittner, R. A., Muckli, L., Waltz, J. A., Kriegeskorte, N., Goebel, R., et al. (2003). Cortical capacity constraints for visual working memory: Dissociation of fMRI load effects in a fronto-parietal network. Neuroimage, 20, $1518-1530$.

Marcantoni, W. S., Lepage, M., Beaudoin, G., Bourgouin, P., \& Richer, F. (2003). Neural correlates of dual task interference in rapid serial visual streams: An fMRI study. Brain and Cognition, 53, 318-321.

Marois, R., Yi, D.-J., \& Chun, M. M. (2004). The neural fate of consciously perceived and missed events in the attentional blink. Neuron, 41, 465-472.

Mayer, J. S., Bittner, R., Linden, D. E. J., \& Nikolić, D. (2007). Attentional demand influences strategies for encoding into visual working memory. Advances in Cognitive Psychology, 3, 429-448.

Mayer, J. S., Bittner, R. A., Nikolic, D., Bledowski, C., Goebel, R., \& Linden, D. E. J. (2007). Common neural substrates for visual working memory and attention. Neuroimage, 36, 441-453.

Mayer, J. S., Roebroeck, A., Maurer, K., \& Linden, D. E. J. (2010). Specialization in the default mode: Task-induced brain deactivations dissociate between visual working memory and attention. Human Brain Mapping, 31, 126-139.

McElree, B., \& Dosher, B. A. (1989). Serial position and set size in short-term memory: The time-course of recognition. Journal of Experimental Psychology: General, 118, 346-373.

Mohr, H. M., Goebel, R., \& Linden, D. E. J. (2006). Contentand task-specific dissociations of frontal activity during maintenance and manipulation in visual working memory. Journal of Neuroscience, 26, 4465-4471.

Oberauer, K. (2002). Access to information in working memory: Exploring the focus of attention. Journal of Experimental Psychology: Learning, Memory, and Cognition, 28, 411-421.

Olivers, C. N. L., \& Nieuwenhuis, S. (2006). The beneficial effects of additional task load, positive affect, and instruction on the attentional blink. Journal of Experimental Psychology: Human Perception and Performance, 32, 364-379.

Owen, A. M., Herrod, N. J., Menon, D. K., Clark, J. C., Downey, S. P., Carpenter, T. A., et al. (1999). Redefining the functional organisation of working memory processes within human lateral prefrontal cortex. European Journal of Neuroscience, 11, 567-574.

Pratt, J., \& Hommel, B. (2003). Symbolic control of visual attention: The role of working memory and attentional control settings. Journal of Experimental Psychology: Human Perception and Performance, 29, 835-845.

Raymond, J. E., Shapiro, K. L., \& Arnell, K. M. (1992). Temporary suppression of visual processing in an RSVP task: An attentional blink? Journal of Experimental Psychology: Human Perception and Performance, 18, 849-860.

Sergent, C., \& Dehaene, S. (2004). Is consciousness a gradual phenomenon? Evidence for an all-or-none bifurcation during the attentional blink. Psychological Science, 15, $720-728$.

Shapiro, K. L., Arnell, K. M., \& Raymond, J. E. (1997). The attentional blink. Trends in Cognitive Sciences, 1 , 291-296.

Shapiro, K. L., Johnston, S. J., Roberts, J. N., \& Zaman, A. (2007). Increased fMRI activity during non-conscious perception in the attentional blink. NeuroReport, 18, 341-345.

Shapiro, K. L., Raymond, J. E., \& Arnell, K. M. (1994). Attention to visual pattern information produces the attentional blink in rapid serial visual presentation. Journal of Experimental Psychology: Human Perception and Performance, 20, 357-371.

Soto, D., Heike, D., Humphreys, G. W., \& Blanco, M. J. (2005). Early, involuntary top-down guidance of attention from working memory. Journal of Experimental Psychology: Human Perception and Performance, 31, 248-261.

Sternberg, S. (1966). High-speed scanning in human memory. Science, 153, 652-654.

Talairach, J., \& Tournoux, P. (1988). Co-planar stereotaxic atlas of the buman brain. Paris: Thieme. 